\title{
Analyse financière de scénarios sylvicoles visant la production de bois d'œuvre de bouleaux jaune et à papier
}

\author{
par Isabelle Legault ${ }^{1}$, Jean-Claude Ruel2,3, Jean-Marie Pouliot ${ }^{2}$ et Robert Beauregard²
}

\section{RÉSUMÉ}

Une analyse de rentabilité financière a été réalisée avec les données forestières d'une strate d'aménagement de la région de La Tuque, au Québec. Les intrants et extrants de deux scénarios sylvicoles adaptés à la sylviculture des bouleaux (Betula alleghaniensis Britton et Betula papyrifera Marsh.), le premier basé sur un régime de coupes progressives et le deuxième, basé sur un régime de jardinage avec régénération par parquets, ont été analysés sur une période de 120 ans. Le logiciel Sylva II a été utilisé pour simuler l'évolution des strates et des produits dans le temps. La rentabilité financière a été évaluée avec le taux interne de rendement et la valeur actualisée nette. Les résultats démontrent que les deux scénarios étudiés sont rentables, le scénario basé sur le jardinage et les parquets étant légèrement plus avantageux que celui faisant intervenir les coupes progressives. De tous les paramètres sylvicoles et financiers évalués, la valeur des produits et le coût des interventions sont les paramètres les plus sensibles. Le scénario d'évolution s'avère être le paramètre le moins sensible. Parmi les paramètres sylvicoles étudiés, la matrice de répartition par produit demeure le plus sensible. Les résultats ne s'appliquent qu'à ce cas précis, mais la méthode utilisée possède l'avantage d'être applicable à une vaste gamme de situations.

Mots clés : bouleau jaune, bouleau à papier, analyse financière, jardinage, coupes progressives

\section{ABSTRACT}

A financial profitability analysis is presented for a particular case study in Québec, using forest data coming from a management stratum located in the region of La Tuque, Quebec. Two silvicultural systems adapted to birch (Betula alleghaniensis Britton and Betula papyrifera Marsh.) regeneration and production, one based on shelterwood cutting and the other on patch cutting combined with single tree selection cutting, are compared over a 120-year period. The Sylva II model has been used to simulate stratum and wood products evolution through time. The financial performance of each scenario is described as the internal rate of return and the net present value. The results demonstrate that both treatments can be profitable and that patch clearcutting combined with the single tree selection cutting system is slightly more profitable than the second system evaluated. The sensitivity analysis shows that, from all criteria considered, treatment costs and product value are the most sensitive parameters. The evolution scenario parameter appears to be the less sensitive one. Finally, the product allocation matrix is the most sensitive of all silvicultural parameters. Although results are relevant for this particular case only, the approach can be broadly applied.

Key words: yellow birch, paper birch, financial analysis, selection cutting, shelterwood cutting

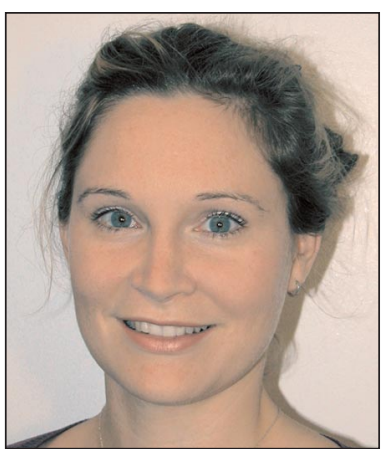

Isabelle Legault

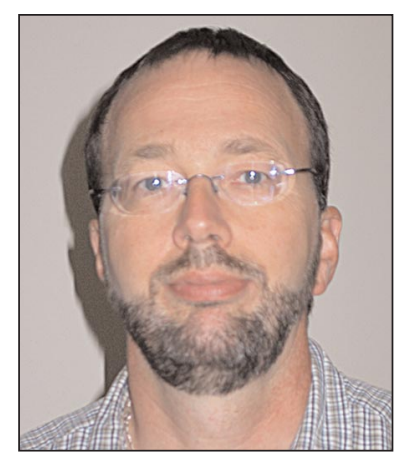

Jean-Claude Ruel

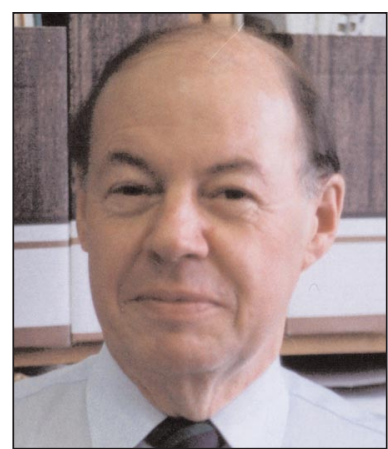

Jean-Marie Pouliot

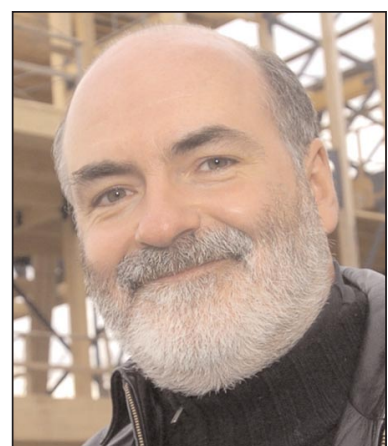

Robert Beauregard

\footnotetext{
${ }^{1}$ Ministère des Ressources Naturelles et de la Faune du Québec.

${ }^{2}$ Département des sciences du bois et de la Forêt, Université Laval, Sainte-Foy, Québec G1K 7P4

${ }^{3}$ courriel : jean-claude.ruel@sbf.ulaval.ca
} 


\section{Introduction}

Dans le contexte de la préparation d'un plan d'aménagement, l'identification du traitement sylvicole approprié pour une strate donnée repose principalement sur l'analyse de l'objectif de production, du site, de la structure et des caractéristiques dendrométriques des peuplements qui la composent. Cependant, la solution pour l'atteinte d'un objectif n'est souvent pas unique en sylviculture (Nyland 1996). Il importe alors de sélectionner, parmi les alternatives acceptables sur les plans écologique et sylvicole, celle permettant une utilisation optimale des fonds limités disponibles pour la sylviculture (Nyland 1996).

Présentement, le jardinage avec régénération par parquets est utilisé par plusieurs industriels du Québec afin de favoriser la régénération des bouleaux (MRN 1998). Ce traitement implique, à tous les 20 ans, une coupe de régénération de superficie de l'ordre de 1 à 2 hectares (parquet) représentant de 15 à $20 \%$ de la superficie originale de la futaie jardinée, ainsi qu'un jardinage par pied d'arbre dans la matrice restante. Théoriquement, l'entière superficie du peuplement aura donc été régénérée en six rotations sur un horizon de 120 ans, ce qui differe grandement de la notion classique de jardinage. Très peu d'informations relatives au parquet, tel qu'on le pratique au Québec, existent dans la littérature et les effets réels de ce traitement pour les strates mixtes sont encore méconnus. De plus, le jardinage par pied d'arbre permet aux essences tolérantes d'atteindre les étages supérieurs mais ne favorise guère les essences à tolérance plus faible, comme le bouleau jaune et encore moins le bouleau à papier (Tubbs et Metzger 1969, LaRocque 1985, Leak et al. 1986). Aussi, de par leur superficie, les parquets favorisent l'établissement d'essences de lumière et de compétition (Roberge 1977, 1979; Walters et Nyland 1989), ce qui peut entraver le développement de bouleaux de qualité (Hannah 1974, Erdman et al. 1981).

La coupe progressive constitue aussi un traitement adapté pour la sylviculture du bouleau jaune (Tubbs et Metzger 1969, Kelty et Nyland 1981, Leak et al. 1986, Hannah 1988, Erdmann 1990) et du bouleau à papier (Perala et Alm 1990). Certains dispositifs installés au Québec témoignent des résultats extrêmement encourageants d'établissement du bouleau dans des sapinières à bouleau jaune à la suite de la coupe d'ensemencement (Malenfant et Patry 2002). Comparativement aux autres procédés de régénération du bouleau jaune et malgré son potentiel prometteur, le procédé de régénération par coupes progressives reste sans doute l'un des moins explorés (Meunier et al. 2002).

L'analyse de rentabilité permet d'éclairer la prise de décision en aménagement forestier (Klemperer 1996). Elle permet ainsi de comparer différentes stratégies sylvicoles et de tenir compte du fait que les coûts et les revenus associés aux traitements different et ne surviennent pas aux mêmes moments (Hanewinkel 2001, Knoke et Plusczyk 2001). Elle permet aussi de tenir compte de l'effet de la sylviculture sur la qualité de la matière ligneuse et l'assortiment de produits qui pourra être obtenu (Anderson et al. 1990, Niese et al. 1995). Puisque les effets à long terme de ces deux traitements demeurent méconnus pour la forêt feuillue et mixte du Québec, la précision sur les estimés de rendement est plutôt faible. Le risque d'erreur est encore plus grand lorsque l'on tente de prédire l'effet de la sylviculture sur la qualité des tiges (Tubbs 1987).
Tableau 1. Caractéristiques dendrométriques de la strate d'aménagement BJ+R C2 70

\begin{tabular}{lccc}
\hline Essences & $\begin{array}{c}\text { Surface } \\
\text { terrière } \\
\left(\mathrm{m}^{2} / \mathrm{ha}\right)\end{array}$ & $\begin{array}{c}\text { Volume } \\
\left(\mathrm{m}^{3} / \mathrm{ha}\right)\end{array}$ & $\begin{array}{c}\text { Volume } \\
\text { de sciage et } \\
\text { déroulage (\%) }\end{array}$ \\
\hline Betula alleghaniensis & 9,1 & 65,0 & 37 \\
Betula papyrifera & 3,4 & 22,5 & 31 \\
Acer saccharum & 2,3 & 16,0 & 37 \\
Acer rubrum & 1,0 & 7,0 & 36 \\
Fraxinus nigra & 0,0 & 0,2 & 0 \\
Populus tremuloides & 1,4 & 13,9 & 70 \\
Abies balsamea & 3,9 & 20,6 & 88 \\
Picea sp. & 1,6 & 13,2 & 88 \\
Thuja occidentalis & 0,2 & 0,9 & 54 \\
\hline Total & 22,9 & 159,4 & 50 \\
\hline
\end{tabular}

La présente étude vise donc à développer une approche méthodologique permettant d'analyser la rentabilité financière de scénarios sylvicoles voués à la production de bouleaux jaune et à papier de qualité sur un horizon de temps couvrant la révolution complète d'un peuplement, c'est-àdire 120 ans. Dans un deuxième temps, nous sommes intéressés à identifier quels critères, autant sylvicoles que financiers, influencent davantage la rentabilité.

\section{Méthodologie \\ Secteur d'étude}

Les données utilisées dans la présente étude proviennent d'une strate d'aménagement de l'aire commune 043-04 située sur les terres publiques $\left(47^{\circ} 43^{\prime} \mathrm{N}, 72^{\circ} 80^{\prime} \mathrm{O}\right)$. Cette dernière se situe au nord-ouest de la ville de La Tuque, dans le sousdomaine de la sapinière à bouleau jaune de l'est (Grondin et al. 1996). Le choix de la région d'étude et de la strate en particulier repose sur la prémisse que l'étude de rentabilité sera davantage utile dans des conditions qui se prêtent à l'application de plusieurs traitements, ce qui survient souvent dans la forêt mixte. De plus, la Mauricie compte 54 millions de mètres cubes de bouleau à papier, ce qui place cette région au premier rang sur le plan de la proportion en volume de cette essence par rapport au volume total toutes essences (Godbout 2002).

L'analyse repose sur une strate mélangée à dominance de bouleau jaune (BJ+R C2 70), pour laquelle un jardinage avec régénération par parquets est prévu immédiatement (Tableau 1). La strate d'aménagement a été retenue comme unité de référence puisqu'il s'agit de celle principalement utilisée dans le cadre du calcul de possibilité forestière au Québec. Il devient donc possible, en simulant cette strate dans le module d'accroissement du logiciel de simulation Sylva II, d'obtenir le niveau de détail (produits par essence $\left(\mathrm{m}^{3} / \mathrm{ha}\right)$ ) nécessaire à l'analyse. Nous nous assurons ainsi que nos hypothèses et nos méthodes de calcul soient cohérentes avec celles utilisées dans le calcul de la possibilité effectué au Québec. Les caractéristiques moyennes de la strate la situent à la limite des seuils décisionnels permettant d'opter pour un traitement de jardinage par parquets ou d'un scénario de coupes progressives d'ensemencement. Elle est aussi très répandue, couvrant 2706 ha de l'aire commune. 


\section{Approche méthodologique}

Un des défis rencontrés dans cette étude était la grande incertitude quant à l'effet à long terme des stratégies sylvicoles. Un choix de base a été fait, à savoir celui de travailler avec les hypothèses utilisées couramment dans la planification forestière et d'évaluer l'impact de l'incertitude qui leur est associée. Dans un premier temps, nous avons élaboré deux scénarios d'aménagement couvrant la vie complète du peuplement. Nous avons ensuite intégré ces scénarios au logiciel Sylva II utilisé pour le calcul de la possibilité forestière sur terre publique. Une analyse de rentabilité a alors pu être réalisée.

A ce stade, la précision de l'estimé de rentabilité demeure largement tributaire des hypothèses utilisées. Reconnaissant leur imprécision, une analyse de sensibilité a été réalisée. Pour ce faire, il a fallu estimer les sources d'incertitude et identifier les paramètres du modèle permettant de les intégrer. On alors pu les faire varier et constater la variation de rentabilité qui en découle.

\section{Élaboration des scénarios sylvicoles}

Deux scénarios sylvicoles appropriés pour la production de bouleaux ont été retenus dans le cadre de cette étude. Le premier scénario (SCJP) repose sur un régime de coupe de jardinage avec parquets et le second (SCPE) est basé sur un régime de coupes progressives. Les deux scénarios (Tableau 2) bâtis autour de ces traitements proposent, dans les deux cas, un scénario intensif de sylviculture avec éclaircie précommerciale et éclaircie commerciale. Un dégagement de bouleaux est prévu dans les parquets afin d'en améliorer la survie, la croissance et la qualité du fut (Hannah 1974, Erdmann et al. 1981).

L'horizon de temps est commun pour les deux scénarios afin d'obtenir une base d'analyse financière comparable (Morissette 1994). Il a été établi d'après l'horizon de temps associé au scénario de jardinage par parquets prescrit par le Ministère des Ressources Naturelles et de la Faune (MRNF), soit 120 ans, et couvre par le fait même la révolution du bouleau jaune. Cet horizon peut sembler long pour une analyse adoptant le point de vue d'un industriel ou d'un propriétaire de boisé. Ce choix est toutefois justifié par la nécessité de tenir compte de l'ensemble des interventions associées à une stratégie sylvicole pour éviter d'introduire un biais initial en faveur d'une stratégie dont les revenus seraient concentrés au début de la période de révolution. Il devient donc possible de comparer l'ensemble des interventions du régime inéquienne (SCJP) à celles du régime équienne (SCPE). Cette méthode est d'ailleurs conforme à celle employée dans d'autres analyses financières comparant deux régimes différents (Hanewinkel 2001, Knoke et Plusczyk 2001).

\section{Données sylvicoles}

Les données nécessaires à l'analyse de rentabilité financière sont des valeurs monétaires associées aux entrées et aux sorties d'argent qui ont lieu à des moments précis dans le temps. Les entrées d'argent étant directement reliées aux volumes récoltés, un défi important a été d'établir les volumes par essence et par produit générés par les différents traitements de chacun des deux scénarios. Le module d'accroissement du Logiciel Sylva II ainsi que les scénarios d'évolution prévus au Manuel d'Aménagement Forestier (MAF) (MRN 1998) ont été utilisés pour générer les volumes
Tableau 2. Scénarios sylvicoles détaillés

\begin{tabular}{|c|c|c|}
\hline Années & $\begin{array}{l}\text { Régime de coupes de } \\
\text { jardinage avec régénération } \\
\text { par parquets (SCJP) }\end{array}$ & $\begin{array}{l}\text { Régime de coupes } \\
\text { progressives } \\
\text { (SCPE) }\end{array}$ \\
\hline 0 & $\begin{array}{l}\text { Jardinage par parquet } 1(\mathrm{P} \# 1) \\
\text { Préparation de terrain }\end{array}$ & $\begin{array}{l}\text { Coupe progressive } \\
\text { d'ensemencement } \\
\text { Débroussaillage } \\
\text { Préparation de terrain }\end{array}$ \\
\hline 5 & Dégagement de P\#1 & \\
\hline 10 & & Coupe finale \\
\hline 20 & $\begin{array}{l}\text { Jardinage par parquet } 2 \\
\text { Préparation de terrain } \\
\text { Éclaircie précommerciale } \mathrm{P} \# 1\end{array}$ & \\
\hline 25 & Dégagement de P\#2 & $\begin{array}{l}\text { Éclaircie } \\
\text { précommerciale }\end{array}$ \\
\hline 40 & $\begin{array}{l}\text { Jardinage par parquet } 3 \\
\text { Préparation de terrain } \\
\text { Éclaircie précommerciale } \mathrm{P} \# 2\end{array}$ & \\
\hline 45 & Dégagement P\#3 & \\
\hline 60 & $\begin{array}{l}\text { Jardinage par parquet } 4 \\
\text { Préparation de terrain } \\
\text { Éclaircie précommerciale } \mathrm{P} \# 3\end{array}$ & \\
\hline 65 & Dégagement P\#4 & \\
\hline 80 & $\begin{array}{l}\text { Jardinage par parquet } 5 \\
\text { Préparation de terrain } \\
\text { Éclaircie précommerciale } \mathrm{P} \# 4 \\
\text { Éclaircie commerciale } \mathrm{P} 11\end{array}$ & Éclaircie commerciale \\
\hline 85 & Dégagement P\#5 & \\
\hline 100 & $\begin{array}{l}\text { Parquet } 6 \\
\text { Préparation de terrain } \\
\text { Éclaircie précommerciale P\#5 } \\
\text { Éclaircie commerciale } 2 \mathrm{P} \# 1 \\
\text { Éclaircie commerciale P\#2 }\end{array}$ & Éclaircie commerciale \\
\hline 105 & Dégagement P\#6 & \\
\hline 120 & $\begin{array}{l}\text { Éclaircie précommerciale P\#6 } \\
\text { Éclaircie commerciale P\#2 } \\
\text { Éclaircie commerciale P\#3 } \\
\text { Coupe finale parquet \#1 }\end{array}$ & Coupe progressive \\
\hline
\end{tabular}

et produits par essence dans le temps, pour chacun des deux scénarios. Nous serons ainsi en mesure d'examiner dans quelle mesure les choix sylvicoles effectués reflètent une logique financière.

La simulation des volumes générés dans chacun des deux scénarios tient compte des données de départ de la strate mais s'effectue quelque peu différemment selon que l'on travaille avec le scénario de coupe progressive ou celui de jardinage par parquets. Dans le cas du jardinage par parquets, le module d'accroissement du logiciel Sylva II permet de simuler 
l'accroissement et les prélèvements de la partie traitée par jardinage. Le choix d'une matrice de répartition par produit à même le logiciel permet de répartir les volumes prélevés entre le déroulage, le sciage, la pâte, la carie et la non-utilisation (les pourcentages associés à chaque produit proviennent d'études de débitage réalisées sur environ 1500 tiges de diamètre et de qualité variable). Selon les normes ministérielles en vigueur en 2003, $20 \%$ de la surface terrière est prélevé par jardinage par pied d'arbre à tous les 20 ans, et sur l'ensemble de la superficie, cinq jardinages auront lieu sur l'horizon de 120 ans. Le module d'accroissement fournit donc à chaque 20 ans les informations relatives aux prélèvements ainsi que les caractéristiques du peuplement résiduel. En appliquant un pourcentage de prélèvement de $16 \%$ (un parquet équivaut à $1 / 6$ d'un ha, soit $16 \%$ ) aux caractéristiques du peuplement résiduel, il est donc possible de connaître les informations associées à la récolte du parquet. Enfin, en ce qui concerne la croissance des essences dans le parquet, les données du scénario d'évolution permettent de générer les volumes par essence et qualité.

Dans le SCPE, le module d'accroissement permet de simuler l'accroissement et les prélèvements entre la coupe d'ensemencement et la coupe finale. Conséquemment, le module n'est utilisé que pour les 10 premières années. Par la suite, le scénario d'évolution comprenant les différents traitements d'éclaircie ainsi que la prochaine coupe progressive est enclenché.

\section{Estimation des revenus et des coûts}

Les revenus s'obtiennent principalement de la valeur des bois produits au cours de chaque intervention. Dans le contexte québécois, des mesures de compensation pour les coûts des interventions sylvicoles sont disponibles, tant sur forêt publique que sur forêt privée. Puisque les montants alloués en forêt privée varient fortement selon les régions et compte tenu que le secteur d'étude se retrouve en forêt publique, on a plutôt ajouté aux revenus le montant accordé par le ministère par le biais des crédits sylvicoles (MRN 2002) (Tableau 3). Puisque les scénarios sont dits intensifs, les traitements incluent toujours les activités de martelage. La valeur des traitements pour lesquels le martelage s'applique est donc majorée de $60 \$$ afin de tenir compte de cette situation. La valeur des produits (Tableau 4) est quant à elle basée sur les listes de prix produites en 2003 par le Syndicat des producteurs de bois de la Mauricie (SPBM 2003) et la Fédération des producteurs de bois du Québec (SPFRQ 2003).

Plusieurs facteurs font varier les coûts, notamment le type de machinerie utilisé, la productivité de la machinerie et de la main d'œuvre, les caractéristiques du peuplement ainsi que les conditions de terrain. Les coûts ont donc été établis en

Tableau 3. Valeur des traitements sylvicoles (MRN 2002)

\begin{tabular}{lc}
\hline Traitements & Valeur $(\$ /$ ha $)$ \\
\hline Préparation de terrain par poquets & 620 \\
Dégagement & 820 \\
Éclaircie précommerciale & 875 \\
Éclaircie commerciale & 660 \\
Jardinage avec régénération par parquets & 305 \\
Coupe progressive & 385 \\
Coupe finale & 325 \\
\hline
\end{tabular}

Tableau 4. Valeur des produits

\begin{tabular}{lccc}
\hline Essence & $\begin{array}{c}\text { Déroulage } \\
\left(\$ / \mathbf{m}^{3}\right)\end{array}$ & $\begin{array}{c}\text { Sciage }^{\mathrm{a}} \\
\left(\$ / \mathbf{m}^{3}\right)\end{array}$ & $\begin{array}{c}\text { Pâte }^{\mathrm{b}} \\
\left(\$ / \mathbf{m}^{3}\right)\end{array}$ \\
\hline Betula alleghaniensis & 272,00 & 75,00 & 36,73 \\
Betula papyrifera Marsh. & 272,00 & 75,00 & 36,73 \\
Acer saccharum & 269,00 & 79,00 & 36,73 \\
Acer rubrum & 0,00 & 48,00 & 36,73 \\
Populus tremuloides & 52,00 & 36,00 & 33,87 \\
Résineux & 0,00 & 59,00 & 51,72 \\
\hline
\end{tabular}

aPrix livré usine

bPrix bord de chemin

consultant des cas concrets et des études déjà réalisées (Del Degan, Massé et Ass. 2002), bien que certaines hypothèses aient dû être posées dans le cas de la coupe progressive d'ensemencement. Ils tiennent compte des coûts d'opération (récolte, tronçonnage, préparation de terrain, etc.), de transport, de supervision et de gestion, d'hébergement et de santé-sécurité, de construction ou d'entretien des chemins, du coût relié à l'inventaire ainsi que celui relié aux autres frais (Tableau 5).

\section{Analyse de rentabilité}

Approche générale

La rentabilité des scénarios est évaluée à l'aide de deux indices, soit le taux interne de rendement et la valeur actuelle nette. L'analyse de sensibilité permet par la suite d'évaluer le risque associé à l'incertitude de chacun des paramètres d'ordres sylvicole et financier.

La valeur actuelle nette (VAN) s'obtient en faisant une prévision des flux monétaires associés aux différentes étapes du projet, en déterminant le taux de rendement minimal acceptable et en actualisant ces flux monétaires (Morissette 1994).

Si la VAN est positive, le projet rapporte plus que le taux d'intérêt guide. Toutes choses étant égales par ailleurs, le projet sera un investissement acceptable. Lorsqu'il s'agit d'évaluer plusieurs projets mutuellement exclusifs, celui affichant la VAN la plus élevée constitue le choix le plus judicieux (Davis et Johnson 1987, Morissette 1994).

$$
\begin{aligned}
& \mathrm{VAN}=\sum_{t=1}^{n} \frac{\mathrm{R}_{t}}{(1+i)^{\mathrm{t}}}-\sum_{t=1}^{n} \frac{\mathrm{C}_{t}}{(1+i)^{\mathrm{t}}} \\
& \begin{array}{l}
\mathrm{R}=\text { Revenus } \\
\mathrm{C}=\text { Coûts } \\
t=\text { année } \\
i=\text { taux d'actualisation }
\end{array}
\end{aligned}
$$

L'utilité du taux interne de rendement (TIR) est reconnue dans le cas d'analyse de projet et notamment dans le cas de projets forestiers (Davis et Johnson 1987). Le taux interne de rendement représente le taux annuel de rendement d'un projet. Celui-ci est obtenu en faisant égaler les revenus et les coûts actualisés, donc il s'agit de trouver le taux d'actualisation permettant d'obtenir une VAN nulle.

$$
\begin{aligned}
& \sum_{t=l}^{n} \frac{\mathrm{R}_{t}}{(1+T R I)^{t}}-\sum_{t=l}^{n} \frac{\mathrm{C}_{t}}{(1+\mathrm{TRI})^{\mathrm{t}}}=0 \\
& \mathrm{R}=\text { Revenus } \\
& \mathrm{C}=\text { Coûts } \\
& t=\text { année }
\end{aligned}
$$




\begin{tabular}{|c|c|c|}
\hline Traitements & Coûts $\left(\$ / \mathrm{m}^{3}\right)$ & Coûts $(\$ / h a)$ \\
\hline \multicolumn{3}{|l|}{ SCPE } \\
\hline Coupe progressive d'ensemencement ${ }^{\mathrm{a}}$ & 67,22 & \\
\hline Coupe finale ${ }^{\mathrm{b}}$ & 54,92 & \\
\hline Débroussaillage/déblaiement/scarifiage & & 1006,04 \\
\hline Éclaircie précommerciale ${ }^{\mathrm{b}}$ & & 1031,59 \\
\hline Éclaircie commerciale $1^{\mathrm{a}}$ & 46,22 & 665,00 \\
\hline Éclaircie commerciale $2^{\mathrm{a}}$ & 39,51 & 665,00 \\
\hline \multicolumn{3}{|l|}{ SCJP } \\
\hline Coupe de jardinage avec régénération par parquets $\# 1^{\mathrm{a}}$ & 65,17 & \\
\hline Coupe de jardinage avec régénération par parquets $\# 2-3-4-5^{b}$ & 58,06 & \\
\hline Préparation de terrain par poquets & & 646,04 \\
\hline Dégagement & & 898,01 \\
\hline Éclaircie précommerciale & & 944,41 \\
\hline Éclaircie commerciale & 37,22 & 665,00 \\
\hline
\end{tabular}

${ }^{a}$ Comprend le coût de construction de chemins

${ }^{b}$ Comprend le coût d'entretien de chemins

Pour les projets mutuellement exclusifs, le projet dont le taux interne de rendement est le plus élevé et supérieur au taux guide, habituellement le rendement obtenu par l'investissement du même montant à la banque, constitue le meilleur choix (Morissette 1994).

L'analyse de sensibilité permet de tenir compte du risque lors des calculs de rentabilité financière (Gregersen et Contreras 1980, Davis et Johnson 1987, Morissette 1994). Dans cette étude, une analyse de sensibilité est réalisée pour différents paramètres financiers et sylvicoles. L'analyse se fait donc pour chaque paramètre d'ordre sylvicole et financier et tient compte des modifications de la VAN engendrées par deux variations, l'une pessimiste et l'autre optimiste. Cette façon de faire, d'ailleurs appliquée dans d'autres analyses de sensibilité de paramètres forestiers (Boltz et al. 2001), est utile dans deux circonstances. Dans un premier temps, il est possible de comparer comment chacun des scénarios réagit aux mêmes variations du paramètre en question et de distinguer dans quel cas le paramètre est plus sensible. Dans un deuxième temps, l'analyse de sensibilité permet d'identifier pour chacun des scénarios quels paramètres ont induit les plus grandes variations, et donc, d'identifier les paramètres les plus sensibles. L'ampleur de la variation des paramètres repose sur une série d'hypothèses basées sur des constatations effectuées sur le terrain ou relatées dans la littérature. Dans d'autres cas, elle est simplement subjective (\% de variation appliqué). La comparaison de la sensibilité des paramètres se fait sur la base du rapport entre la variation de VAN et celle du paramètre, toutes deux exprimées en pourcentage.

Afin de sélectionner les paramètres sylvicoles à inclure dans l'étude de sensibilité, nous avons identifié les sources d'incertitude quant aux effets de l'utilisation des parquets, du jardinage par pied d'arbre et de la coupe progressive sur l'établissement et le développement des nouveaux peuplements. Les principales sources d'incertitude identifiées concernent principalement le succès de l'établissement des essences désirées suite aux coupes de régénération, la possibilité de retards de croissance et de croissance plus faible des essences désirées dans les parquets et dans les coupes de jardinage, la composition en essences très variable des peuplements matures obtenus suite à l'application des divers traitements, et finalement, le type de produit et la quantité retrouvés dans les peuplements issus des divers traitements.

Dans un deuxième temps, un parallèle a été fait entre les éléments sylvicoles identifiés précédemment et les paramètres de simulation utilisés dans le logiciel Sylva II. Dans le module d'accroissement de Sylva II, la croissance des essences est considérée par l'utilisation de deux paramètres : les taux de passage et les zones d'accroissement (MRN 1999). La zone d'accroissement caractérise l'accroissement annuel moyen des tiges des différentes essences et se choisit en fonction de la localisation géographique du peuplement à travers le Québec. Les taux de croissance quant à eux se choisissent en fonction du type d'intervention qui a eu lieu dans le peuplement, de la densité et du type du peuplement ainsi que de la mortalité qui afflige le peuplement. Le type de produit obtenu par essence se traite grâce aux matrices de répartition par produit. Quant à la composition en essences et en produits des peuplements prématures et matures issus de coupes de régénération, on doit se référer au scénario d'évolution présent dans le logiciel.

L'identification de ces paramètres permet conséquemment de tenir compte des sources d'incertitude qui sont évaluées et quantifiées par l'analyse de sensibilité. De façon générale, les valeurs de départ propres à chacun des paramètres sylvicoles sont conformes aux hypothèses d'aménagement prévues au MAF ainsi qu'aux hypothèses relatives au calcul de possibilité. L'ensemble de ces paramètres permet de définir pour chacun des deux scénarios une simulation considérée comme «témoin». Les taux de passage utilisés pour la simulation témoin sont les taux moyens recommandés par le Ministère des Ressources Naturelles et de la Faune du Québec (MRNF) (François Pelletier, communication personnelle). Bien que la zone d'accroissement de l'aire commune soit la zone 25, c'est la zone d'accroissement 17 qui a été sélectionnée dans le cadre des simulations (MRN 1999, voir Annexe 3). Ces deux zones sont contiguës, la zone 17 présentant les meilleures conditions de croissance. Comme la strate possède une bonne proportion d'érable à sucre et d'érable rouge, la zone 17 a été privilégiée par rapport à la zone 25 . De plus, outre pour le 
peuplier faux-tremble, les courbes de croissance des feuillus de ces deux zones sont identiques. On note cependant quelques différences de croissance des résineux. Finalement, la matrice intitulée «standard nord» a été utilisée comme matrice de répartition par produit de départ (MRN 1999) et le scénario d'évolution de départ retenu est le scénario 14.2 (MRN 1998)

Le taux d'actualisation, les coûts des traitements et des opérations, ainsi que la valeur des produits influencent la rentabilité des scénarios sylvicoles. Dans notre analyse, un taux d'actualisation de $4 \%$ est employé. Ce dernier correspond au taux fréquemment utilisé dans le cadre des projets forestiers (Davis et Johnson 1987) et des projets environnementaux de longue durée (Weitzman 2001).

\section{Variation des paramètres sylvicoles}

Les taux de base utilisés pour la simulation témoin se basent sur les recommandations du MRNF (Lessard et al. 2004). Ainsi, les taux naturels de croissance ont été utilisés pour la première période de simulation. Cette série de taux représente les plus faibles accroissements qu'il soit possible d'utiliser dans le module de simulation Sylva II. Une fois un premier traitement sylvicole appliqué, on recommande alors d'utiliser la série de taux nommée $\mathrm{ABCD}$ avec mortalité. Cette série de taux est dite améliorée et tient compte de la mortalité dans chacune des classes de diamètre. Afin de simuler une situation pessimiste, nous avons choisi de conserver le taux naturel tout au long de l'horizon de simulation. Cette façon de faire pourrait sensiblement reproduire certains résultats observés à la suite de coupes de jardinage opérationnelles (Bédard et Brassard 2002). À l'opposé, dans le cas de la situation optimiste, nous avons substitué le taux amélioré par le taux présentant les plus forts accroissements, soit le taux CD sans mortalité. En effet, cette série de taux simule des accroissements de peuplements très ouverts où aucune mortalité ne survient.

La zone d'accroissement 17 sert de zone de base témoin dans chacun des deux scénarios. Pour les fins de l'analyse, deux nouvelles zones ont été créées afin de reproduire une situation pessimiste et une seconde optimiste. Dans la situation pessimiste, nous avons sélectionné pour chacune des essences les accroissements les plus faibles, alors que dans la situation optimiste, les accroissements les plus forts ont été choisis. Les modifications touchent seulement les accroissements des deux essences majoritaires, soit le bouleau jaune et le bouleau à papier, afin de tenir compte du ralentissement de croissance possible causé par la présence importante des espèces de compétition au cours de 20 premières années (Archambault et al. 1997). Les essences résineuses et l'érable à sucre étant plus tolérants et donc moins affectés par la compétition importante, aucune variation des courbes n'a été effectuée pour ces espèces.

Dans le cadre de l'étude de sensibilité, deux nouvelles matrices de répartition par produit ont été créées. Dans la première, les volumes de déroulage et de sciage pour l'ensemble des essences ont été augmentés de $25 \%$ par rapport à la matrice de départ. Les volumes de pâte, de carie et de nonutilisation ont été diminués de façon à ne pas modifier le volume total. Cette situation très optimiste n'est pas le reflet de la réalité mais permet d'évaluer l'impact d'une augmentation de la qualité issue d'un aménagement intensif. Les volumes de sciage et de déroulage de la deuxième matrice ont quant à eux été diminués de $45 \%$ afin de représenter une situation pessimiste. Bien que ce choix revête un certain caractère arbitraire, il tente de reproduire la qualité des volumes de bois observés à la cour de l'usine (Christian Picard, ingénieur forestier, Commonwealth Plywood Co. Ltd., communication personnelle).

Deux éléments intrinsèques au scénario d'évolution ont été vérifiés par l'analyse de sensibilité : la répartition des volumes par essence et la répartition des volumes par type de produit. En ce qui concerne l'analyse de la répartition des volumes par essence, nous avons premièrement considéré l'effet probable d'une grande ouverture (parquet) sur l'évolution des essences dans le temps. En accord avec la littérature (Marquis 1967, Boivin 1971, Roberge 1977), nous avons modifié le scénario afin d'augmenter les volumes associés aux essences intolérantes et diminuer ceux des essences tolérantes à intermédiaires. Nous avons également diminué les volumes des résineux (Archambault et al. 1997). De l'autre côté, nous avons évalué l'effet d'une augmentation des essences tolérantes à intermédiaires et des résineux ainsi qu'une diminution des essences intolérantes afin de considérer l'augmentation de l'installation des essences plus tolérantes à l'ombre favorisée par la coupe d'ensemencement. Dans l'ensemble des cas, la diminution est simulée par une perte de $1 \%$ des volumes d'une essence pour toutes les qualités, et l'augmentation, par un gain de volume de $1 \%$.

En ce qui concerne l'analyse de la répartition des volumes par produit, nous avons testé l'effet d'une diminution et d'une augmentation globale de la qualité des tiges dans les scénarios. En effet, il est raisonnable de penser que l'application d'une petite coupe à blanc puisse diminuer la quantité de tiges de qualité (Marquis 1967) et qu'à l'inverse, la coupe progressive puisse l'augmenter (Leak et al. 1986). La diminution de qualité est simulée par une baisse de $1 \%$ des volumes de sciage de chaque essence de la classe ainsi qu'une augmentation de $1 \%$ de la pâte, aucun déroulage n'étant prévu au scénario original. Dans le cas de l'augmentation de qualité, $1 \%$ du volume original du bouleau jaune et de l'érable à sucre est redistribué au déroulage alors que la pâte est diminuée de cette même quantité. Pour les autres essences feuillues, le sciage est majoré de $1 \%$ tandis que la pâte est diminuée selon les mêmes proportions. Aucun changement n'est effectué pour les résineux puisque la totalité du volume se retrouve déjà en sciage.

\section{Variation des paramètres financiers}

Le choix d'un taux d'intérêt adéquat constitue une étape critique de l'analyse de rentabilité. Dans un premier temps, afin de rester à une échelle représentative de la réalité, nous avons évalué la sensibilité de nos deux scénarios à une faible variation du taux d'actualisation. Une variation de plus ou moins $1 \%$ a donc été appliquée au taux de départ de $4 \%$. Dans un deuxième temps, nous avons considéré un taux déclinant tel que recommandé par Weitzman (2001). Dans le cadre d'analyse de projets d'ordre environnemental, les intrants et extrants sont évalués sur des dizaines, voire des centaines d'années. Lorsque l'horizon de temps est long, l'utilisation d'un taux d'intérêt constant fait en sorte que l'actualisation des revenus et des coûts survenant dans un futur de plus en plus éloigné explique de moins en moins la rentabilité ou la non-rentabilité du projet en question. Comparé à un projet de courte durée, aucun projet 
environnemental n'est donc financièrement plus avantageux du seul fait de sa durée. Devant cette situation, plusieurs économistes se questionnent sur la pertinence d'utiliser un taux constant et à ce jour, aucun consensus n'existe sur l'utilisation d'un taux mieux adapté. Pour palier ce problème, Weitzman (2001) propose d'utiliser un taux d'intérêt déclinant en fonction de l'horizon de simulation. Nous avons ainsi examiné l'impact de cette approche en utilisant un taux de $4 \%$ pour les 5 premières années, $3 \%$ de 6 à 25 ans, $2 \%$ entre 26 et 75 ans et $1 \%$ pour le reste de l'horizon de simulation.

La valeur des produits est susceptible de varier grandement dans le temps pour plusieurs raisons. Encore une fois, des variations de $1 \%$ en plus et en moins ont été appliquées afin de créer les situations optimistes et pessimistes. Dans chaque cas, la variation touche l'ensemble des produits pour l'ensemble des essences.

Le coût des opérations est également voué au changement. L'amélioration des techniques de coupe, l'optimisation de la machinerie, la rareté de la main-d'œuvre, la fluctuation du coût de l'essence sont autant de facteurs qui influencent le coût de la récolte. La même procédure que celle utilisée pour évaluer la sensibilité des produits est appliquée pour évaluer la sensibilité de ce paramètre, soit des variations de $1 \%$.

\section{Résultats et Discussion}

\section{Rentabilité des scénarios sylvicoles}

L'analyse des figures 1 et 2 permet d'affirmer qu'en utilisant les hypothèses et les rendements inclus au MAF et un taux d'actualisation de $4 \%$, toutes choses étant égales par ailleurs, le SCJP est très légèrement supérieur au SCPE en terme de rentabilité. Bien que la différence de la VAN soit faible entre les deux scénarios, le TIR nettement plus important pour le SCJP montre que ce scénario représente un investissement plus avantageux. Une étude similaire comparant huit traitements sylvicoles pour une strate feuillue du Wisconsin (Niese et Strong 1992) arrive à la même conclusion.

Plusieurs facteurs peuvent expliquer que le SCJP soit plus rentable que le SCPE. Le principal est certainement lié au moment des entrées et des sorties d'argent dans le temps, jumelé à la rentabilité de la première intervention. Cette réalité économique propre à la comparaison de régimes équiennes et inéquiennes est bien documentée (Hanewinkel 2001, Knoke et Plusczyk 2001). Le processus d'actualisation fait en sorte que les premières entrées ou sorties de fonds occupent un poids plus important dans le calcul de VAN, tandis que, plus on avance dans le temps, moins les entrées et sorties de fonds jouent un rôle important. Conséquemment, comme la première intervention est déficitaire d'environ 630 $\$$ en raison du coût élevé des travaux et de la construction de chemin, les entrées de fonds subséquentes n'arrivent tout simplement pas à rendre la VAN du SCPE supérieure à celle du SCJP, malgré que le flux monétaire de la deuxième intervention du SCPE soit pratiquement 4 fois plus élevé que la deuxième intervention de la SCJP. Un rapprochement peut également être fait avec deux études comparant l'avantage financier de transformer un peuplement équienne en peuplement inéquienne (Hanewinkel 2001, Knoke et Plusczyk 2001). Celles-ci démontrent justement qu'il y a peu de différence d'un point de vue strictement financier entre les deux régimes.

Par ailleurs, lorsque les deux scénarios de cette présente étude sont comparés, on remarque que la première intervention est déficitaire. Les coûts élevés associés à la construction de chemin (et non à l'entretien et à la réfection lors des interventions subséquentes) expliquent cette situation. En effet, en faisant l'exercice de ne pas considérer les coûts de construction, mais seulement ceux de l'entretien pour la première intervention, on remarque que la rentabilité et le taux de rendement interne des deux scénarios varie énormément (Figures 3 et 4). Dans un contexte d'aménagement intensif, la présence d'un réseau routier bien développé et la proximité des aires de coupe aux usines sont nécessaires. Il n'est pas rare, non plus, d'intensifier la sylviculture dans les peuplements de fort potentiel situés là où le réseau routier est déjà bien établi. Dans cette perspective, les coûts de construction de chemin ne devraient pas être considérés dans l'analyse financière, mais bien seulement ceux d'entretien et de réfection. Même en ne considérant qu'une réfection des chemins à la première intervention, le SCPE demeure toujours légèrement moins rentable que son vis-à-vis. Le gain monétaire engendré ne suffit pas à rendre la première intervention du SCPE rentable comme c'est le cas dans le

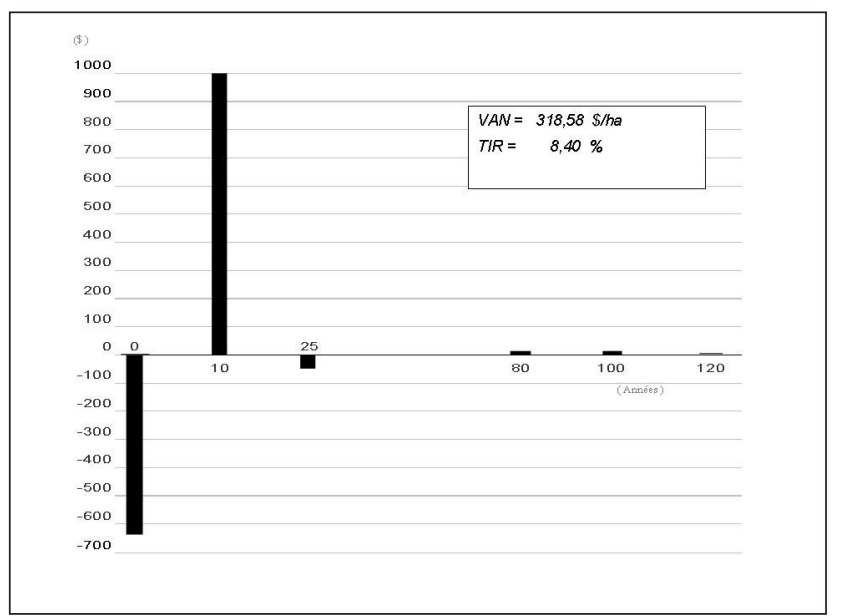

Fig. 1. Diagramme des flux monétaires nets actualisés pour le scénario de coupes progressives, données de base (simulation témoin]

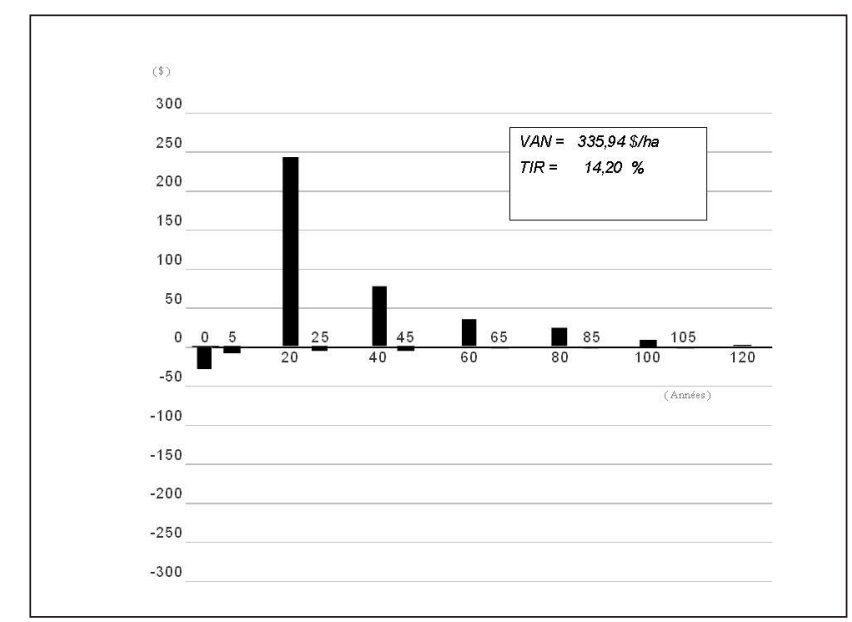

Fig. 2. Diagramme des flux monétaires nets actualisés pour le scénario de jardinage avec régénération par parquets, données de base (simulation témoin) 


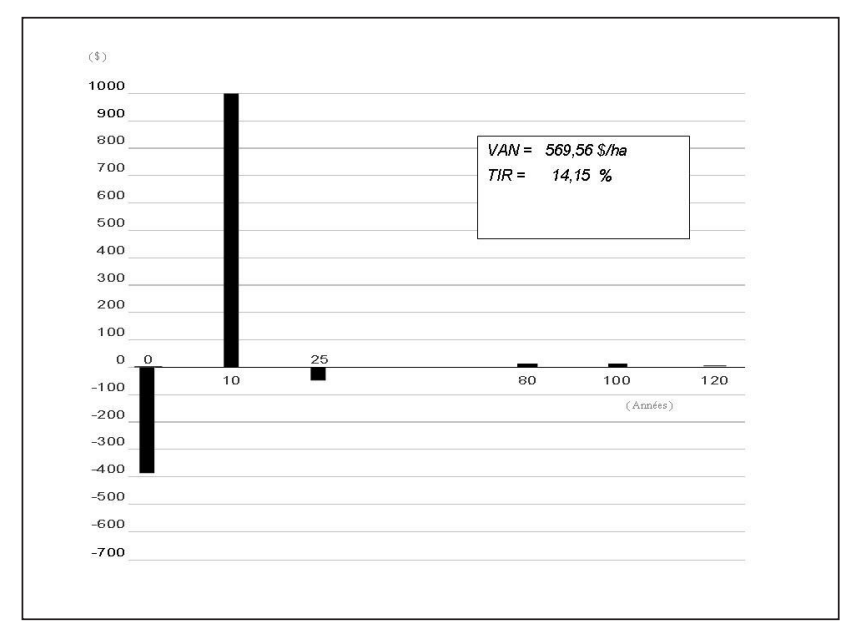

Fig. 3. Diagramme des flux monétaires nets actualisés pour le scénario de coupe progressive, sans construction de chemin 1e intervention

SCJP. C'est donc dire que les coûts élevés de la coupe d'ensemencement jouent un rôle très important dans la rentabilité du scénario. Il est important de mentionner que le coût considéré pour la coupe d'ensemencement est élevé puisqu'il provient d'une étude réalisée dans un cadre expérimental (Meunier et al. 2002). L'amélioration des techniques utilisées, et l'utilisation à plus grande échelle ne peuvent que faire diminuer le coût du traitement d'ici les prochaines années. Dans cette perspective, la VAN du scénario SCPE est susceptible d'augmenter changeant ainsi les conclusions de la présente étude.

\section{Analyse de sensibilité}

Les résultats de l'analyse de rentabilité se retrouvent aux Tableaux 6 et 7. Les Tableaux 8 et 9 montrent quant à eux la sensibilité de chacun des paramètres étudiés.

\section{Taux de passage}

Les résultats des Tableaux 6 et 8 démontrent donc que les taux de passage constituent un paramètre plus sensible dans le cas du SCJP que dans le cas du SCPE, comme en témoignent les rapports de variation VAN/paramètre plus importants autant pour les situations pessimistes et optimistes dans le cas du SCJP. Ce résultat est tout à fait logique puisque l'intervention des taux de passage dans chacun des scénarios differe. En effet, la série de taux de passage a un effet sur l'ensemble de l'horizon de temps du SCJP étant donné qu'un couvert résiduel est présent jusqu'à la cent vingtième année. Bien que le couvert résiduel décroisse en superficie et en volume de période en période, il n'en reste pas moins que le scénario subit l'effet d'une baisse ou d'une hausse beaucoup plus fortement que le SCPE qui lui, n'est affecté par le taux de passage que pour une période de 10 ans.

Lorsqu'on compare les VAN des deux scénarios entre elles, on remarque que, dans la situation pessimiste, le SCPE devient plus rentable que le SCJP. Dans le scénario optimiste, c'est l'inverse qui se produit et la VAN du SCJP surpasse celle du SCPE. Conséquemment, dans le cas où les taux de croissance du peuplement sur pied sont plus faibles que ceux estimés, l'adoption d'un scénario basé sur les coupes progressives devient plus avantageuse d'un point de vue

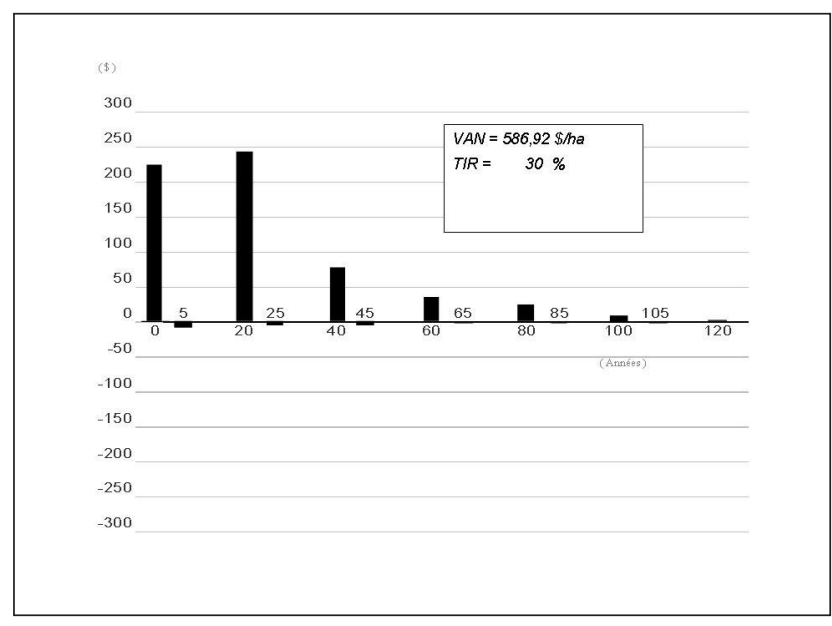

Fig. 1. Diagramme des flux monétaires nets actualisés pour le scénario de coupes progressives, données de base (simulation témoinJFig. 4. Diagramme des flux monétaires nets actualisés pour le scénario de jardinage avec régénération par parquets, sans construction de chemin $1^{\mathrm{e}}$ intervention

monétaire. Dans les conditions typiques du Québec, il semble peu probable qu'un peuplement traité par jardinage par pied d'arbre puisse afficher des taux de croissance similaires à des peuplements ouverts (densité $\mathrm{C}$ et $\mathrm{D}$ ) non affectés par la mortalité (Bédard et Brassard 2002). Par contre, il est possible de penser que les taux d'accroissement du peuplement résiduel ayant subi une coupe progressive d'ensemencement de l'ordre de $45 \%$ d'ouverture puissent se rapprocher de cette série de taux plus optimistes. La comparaison de nos deux scénarios témoins ne reflète donc pas la réalité puisque nous avons utilisé pour les deux scénarios les mêmes séries de taux d'accroissement. Conséquemment, pour rester fidèle à la réalité, il faudrait considérer pour le SCPE des taux de passage se situant quelque part entre le taux témoin $(\mathrm{ABCD}$ avec mortalité) et le taux optimiste (CD sans mortalité) alors qu'il faudrait considérer pour le SCJP, des taux se rapprochant du taux naturel. Dans ces conditions, l'écart de la VAN augmente entre les deux scénarios et le SCPE devient le choix le plus financièrement avantageux.

\section{Zone d'accroissement}

Tout comme dans le cas précédent, l'ampleur des variations de la VAN et du TIR est à nouveau plus importante dans le cas du SCJP que dans celui du SCPE (Tableau 6). L'examen des rapports de sensibilité (Tableau 8) confirme que la zone d'accroissement est plus sensible dans le cas du SCJP que dans le cas du SCPE. L'intervention des zones d'accroissement à chacune des périodes de l'horizon de simulation dans le cas du SCJP comparativement à une intervention limitée aux deux premières périodes dans le cas du SCPE explique la différence.

Lorsqu'on exprime les variations en pourcentage (Tableau 8), on s'aperçoit que, dans tous les cas, la sensibilité obtenue est beaucoup plus faible que celle obtenue avec le paramètre précédent. On remarque aussi que, dans les situations pessimistes, le rapport de sensibilité est plus élevé que dans la situation optimiste pour chacun des deux scénarios. Il serait possible de penser que chacun des scénarios est plus sensible à des diminutions de croissance en diamètre. Malgré tout, comme la détermination du pourcentage de variation du 
Tableau 6. Rentabilité et rendements des scénarios en fonction des variations des différents paramètres sylvicoles

\begin{tabular}{|c|c|c|c|c|c|c|c|}
\hline \multirow[b]{2}{*}{ Paramètres } & \multicolumn{4}{|c|}{$\operatorname{VAN}^{\mathrm{a}}(\$ / \mathrm{ha})$} & \multicolumn{3}{|c|}{$\operatorname{TIR}^{\mathrm{b}}(\%)$} \\
\hline & Scénarios & Pessimiste & Témoin & Optimiste & Pessimiste & Témoin & Optimiste \\
\hline \multirow[t]{2}{*}{ Taux de passage } & SCPE & 206 & 319 & 411 & 7,0 & 8,4 & 9,43 \\
\hline & SCJP & 190 & 336 & 532 & 12,3 & 14,2 & 15,6 \\
\hline \multirow[t]{2}{*}{ Zone d'accroissement } & SCPE & 282 & 319 & 342 & 8,0 & 8,4 & 8,7 \\
\hline & SCJP & 261 & 336 & 384 & 13,2 & 14,2 & 14,7 \\
\hline \multirow[t]{2}{*}{ Matrice de répartition par produits } & SCPE & -599 & 319 & 773 & 0,7 & 8,4 & 14,8 \\
\hline & SCJP & -405 & 336 & 718 & 0,57 & 14,2 & 38,0 \\
\hline \multirow[t]{2}{*}{ Scénario d'évolution (volume/essence) } & SCPE & 318 & 319 & 319 & 8,4 & 8,4 & 8,4 \\
\hline & SCJP & 336 & 336 & 336 & 14,2 & 14,2 & 14,2 \\
\hline \multirow[t]{2}{*}{ Scénario d'évolution (volume/produit) } & SCPE & 318 & 319 & 321 & 8,4 & 8,4 & 8,4 \\
\hline & SCJP & 336 & 336 & 336 & 14,2 & 14,2 & 14,2 \\
\hline
\end{tabular}

${ }^{\mathrm{a}}$ valeur actuelle nette

$b_{\text {taux interne de rendement }}$

Tableau 7. Rentabilité et rendements des scénarios en fonction des variations des différents paramètres financiers

\begin{tabular}{|c|c|c|c|c|c|c|c|c|}
\hline \multirow[b]{2}{*}{ Paramètres } & \multirow[b]{2}{*}{ Scénarios } & \multicolumn{4}{|c|}{$\operatorname{VAN}^{\mathrm{a}}(\$ / \mathrm{ha})$} & \multicolumn{3}{|c|}{$\operatorname{TIR}^{\mathrm{b}}(\%)$} \\
\hline & & Pessimiste & Témoin & Optimiste & Décroissant & Pessimiste & Témoin & Optimiste \\
\hline \multirow[t]{2}{*}{ Taux d'actualisation } & SCPE & 230 & 319 & 430 & 668 & - & - & - \\
\hline & SCJP & 238 & 336 & 494 & 1004 & - & - & - \\
\hline \multirow[t]{2}{*}{ Valeur des produits } & SCPE & 244 & 319 & 393 & - & 7,35 & 8,4 & 9,5 \\
\hline & SCJP & 281 & 336 & 391 & - & 10,7 & 14,2 & 27,0 \\
\hline \multirow[t]{2}{*}{ Coûts des interventions } & SCPE & 239 & 319 & 399 & - & 7,2 & 8,4 & 9,7 \\
\hline & SCJP & 273 & 336 & 398 & - & 10,3 & 14,2 & 34 \\
\hline
\end{tabular}

avaleur actuelle nette

btaux interne de rendement

Tableau 8. Sensibilité des différents paramètres sylvicoles dans chacun des scénarios

\begin{tabular}{|c|c|c|c|c|c|c|c|}
\hline \multirow[b]{2}{*}{ Paramètres } & \multirow[b]{2}{*}{ Scénarios } & \multicolumn{3}{|c|}{ Pessimiste } & \multicolumn{3}{|c|}{ Optimiste } \\
\hline & & $\begin{array}{c}\text { Variation } \\
\text { de la } \\
\text { VAN }^{\mathrm{a}} \\
(\%)\end{array}$ & $\begin{array}{l}\text { Variation } \\
\text { du } \\
\text { paramètre } \\
(\%)\end{array}$ & Rapport & $\begin{array}{c}\text { Variation } \\
\text { de la } \\
\text { VAN }^{\mathrm{a}} \\
(\%)\end{array}$ & $\begin{array}{l}\text { Variation } \\
\text { du } \\
\text { paramètre } \\
(\%)\end{array}$ & Rapport \\
\hline \multirow[t]{2}{*}{ Taux de passage } & SCPE & $-35,4$ & $-11,0$ & 3,2 & 29,0 & 15,4 & 1,9 \\
\hline & SCJP & $-43,5$ & $-11,0$ & 4 & 58,3 & 15,4 & 3,8 \\
\hline \multirow{2}{*}{ Zone d'accroissement } & SCPE & $-11,0$ & $-10,2$ & 1,1 & 8,0 & 16,9 & 0,5 \\
\hline & SCJP & $-22,0$ & $-10,2$ & 2,2 & 14,0 & 16,9 & 0,8 \\
\hline \multirow[t]{2}{*}{ Matrice de répartition par produits } & SCPE & $-88,0$ & $-45,0$ & 2,0 & 142,7 & 25,0 & 5,7 \\
\hline & SCJP & $-20,6$ & $-45,0$ & 0,5 & 113,6 & 25,0 & 4,5 \\
\hline \multirow[t]{2}{*}{ Scénario d'évolution (volume/essence) } & SCPE & $-0,2$ & $-1,0$ & 0,2 & 0,0 & 1,0 & 0,0 \\
\hline & SCJP & $-0,2$ & $-1,0$ & 0,2 & 0,0 & 1,0 & 0,0 \\
\hline \multirow[t]{2}{*}{ Scénario d'évolution (volume/produit) } & SCPE & $-0,2$ & $-1,0$ & 0,2 & 0,0 & 1,0 & 0,0 \\
\hline & SCJP & 0,9 & 1,0 & 0,9 & 0,1 & 1,0 & 0,1 \\
\hline
\end{tabular}

${ }^{\mathrm{a}}$ valeur actuelle nette 


\begin{tabular}{|c|c|c|c|c|c|c|c|}
\hline \multirow[b]{2}{*}{ Paramètres } & \multirow[b]{2}{*}{ Scénarios } & \multicolumn{3}{|c|}{ Pessimiste } & \multicolumn{3}{|c|}{ Optimiste } \\
\hline & & $\begin{array}{c}\text { Variation } \\
\text { de la } \\
\text { VAN }^{\mathrm{a}} \\
(\%)\end{array}$ & $\begin{array}{l}\text { Variation } \\
\text { du } \\
\text { paramètre } \\
(\%)\end{array}$ & Rapport & $\begin{array}{c}\text { Variation } \\
\text { de la } \\
\text { VANa } \\
(\%)\end{array}$ & $\begin{array}{l}\text { Variation } \\
\text { du } \\
\text { paramètre } \\
(\%)\end{array}$ & Rapport \\
\hline \multirow[t]{2}{*}{ Taux d'actualisation } & SCPE & $-28,0$ & $-25,0$ & 1,1 & 35,0 & 25,0 & 1,4 \\
\hline & SCJP & $-29,3$ & $-25,0$ & 1,2 & 47,1 & 25,0 & 1,9 \\
\hline \multirow[t]{2}{*}{ Valeur des produits } & SCPE & $-23,5$ & $-1,0$ & 23,5 & 23,5 & 1,0 & 23,5 \\
\hline & SCJP & $-16,3$ & $-1,0$ & 16,3 & 16,3 & 1,0 & 16,3 \\
\hline \multirow[t]{2}{*}{ Coûts des interventions } & SCPE & $-25,1$ & $-1,0$ & 25,1 & 25,1 & 1,0 & 25,1 \\
\hline & SCJP & $-18,6$ & $-1,0$ & 18,6 & 18,6 & 1,0 & 18,6 \\
\hline
\end{tabular}

paramètre ne considère que l'essence principale, il faut rester prudent dans les conclusions tirées.

Dans la situation pessimiste, le SCPE présente une VAN plus élevée que son comparable. Par contre, la valeur du TIR pour le SCJP est supérieure à celle du SCPE en situation pessimiste. La stratégie à privilégier dépendra du critère de rentabilité sélectionné lorsque l'accroissement en diamètre est plus faible que ce qui était anticipé. À l'inverse, si nous croyons que la zone d'accroissement doit être plus élevée, alors le SCJP devrait être privilégié.

\section{Matrice de répartition par produit}

La variation des matrices de répartition par produit a entraîné des écarts importants de rentabilité pour les deux scénarios. D'ailleurs, à un taux d'actualisation de $4 \%$, la matrice de répartition par produit pessimiste engendre la non rentabilité des deux scénarios sylvicoles et un taux de rendement interne légèrement supérieur à 0 (Tableau 6).

Contrairement aux tests réalisés avec les deux paramètres précédents, l'ampleur de la variation de la VAN est plus importante dans le cas du SCPE que dans celui du SCJP. De ce fait, la diminution de la qualité du sciage et du déroulage (situation pessimiste) implique que le VAN du SCJP est plus élevée que celle du SCPE. Â l'inverse, l'augmentation de la qualité du sciage et du déroulage induit une différence positive de la VAN en faveur du SCPE. Cette réaction est tout à fait normale puisque l'impact des variations n'est pas pris en compte aux mêmes moments dans chacun des deux scénarios. Comme nous l'avons déjà mentionné auparavant, les traitements générant les principaux volumes et donc les entrées d'argent les plus importantes, surviennent aux années 0 et 10 dans le cas du SCPE. Si nous comparons les volumes obtenus au cours de ces 10 premières années dans chacun des deux scénarios, on remarque que ceux du SCPE sont plus élevés. Conséquemment, l'impact d'une matrice de répartition différente est plus marqué pour le SCPE que le SCJP en raison des volumes supérieurs obtenus en début de période.

Un autre fait important à remarquer est le taux interne de rendement obtenu dans la situation optimiste du SCJP. On retrouve dans le Tableau 6 une valeur de $38 \%$, mais en fait, le taux interne de rendement ne peut jamais être atteint complètement puisque la VAN n'égalise jamais 0 . Cette situation particulière s'explique simplement par le fait que l'augmentation de la qualité des tiges récoltées lors de chaque intervention est telle que la valeur des produits obtenue est toujours supérieure aux coûts d'opérations et de récolte. Chaque intervention de récolte au cours de l'horizon de simulation est donc rentable. Cette situation ne se reproduit pas dans le cas du SCPE puisque, malgré l'augmentation de la qualité des tiges et de la valeur totale des produits obtenue, les coûts de la coupe d'ensemencement demeurent toujours plus élevés que les revenus. Cette intervention positionnée au début de l'horizon de simulation a alors un impact déterminant sur le TIR.

On remarque aussi que les rapports de sensibilité sont plus importants dans le cas du SCPE que dans celui du SCJP (Tableau 8). De plus, les résultats démontrent que la sévérité du rapport differe selon que la situation soit pessimiste ou optimiste. En effet, une augmentation de la qualité des produits engendre une plus grande variation que ne le fait une diminution. Cette situation démontre donc que la relation qui existe entre les scénarios (SCJP et SCPE) et les matrices de répartition par produit n'est pas directement proportionnelle.

\section{Scénario d'évolution}

Les résultats démontrent que le scénario d'évolution n'est aucunement sensible, autant pour le SCPE que pour le SCJP (Tableaux 6 et 8). La minime différence de variation entre les deux scénarios est due au fait que le scénario d'évolution ne s'applique qu'à la superficie traitée antérieurement par parquet (1/6 de la superficie de la strate) dans le cas du SCJP et à l'ensemble de la strate dans le cas du SCPE. Malgré tout, on remarque que les rapports (Tableau 8) sont extrêmement faibles dans tous les cas. Cette affirmation est vraie autant pour la modification des volumes par essences dans le scénario que la modification des produits par essence. L'implication tardive du scénario d'évolution dans l'horizon de simulation pour chacun des deux scénarios explique pourquoi ce paramètre est peu sensible. En effet, dans les deux scénarios, les premières entrées et sorties d'argent associées à la récolte de volumes issus du scénario d'évolution ne surviennent qu'à la $80^{\mathrm{e}}$ année. Avec le processus d'actualisation, ces flux monétaires n'occupent que très peu de poids dans le calcul de la VAN.

\section{Taux d'actualisation}

La modification du taux d'actualisation influence à la fois le SCJP et le SCPE, bien que la VAN du SCJP varie légèrement davantage que celle du SCPE (Tableau 9). Bien entendu, la 
modification du taux d'actualisation n'affecte en rien le taux interne de rendement des scénarios puisque aucune valeur de flux monétaire dans le temps n'est modifiée. Seule la valeur actualisée nette de ces flux change. Ces constatations se reflètent également dans les rapports de sensibilité présentés au Tableau 9. On remarque aussi que la différence entre les rapports issus des situations optimistes est plus importante que celle observée entre les rapports issus des situations pessimistes. Encore une fois, une relation non directement proportionnelle entre les scénarios et le paramètre explique ce phénomène.

Bien qu'il ne fasse pas partie de l'analyse de sensibilité, l'emploi du taux déclinant démontre qu'en accordant davantage de poids aux flux monétaires survenant tard dans l'horizon de simulation, le SCJP devient alors plus rentable. En effet, la VAN de ce scénario est pratiquement 3 fois plus élevée que dans le cas témoin. Parallèlement, le SCPE réagit également positivement à l'utilisation d'un taux déclinant, cependant, l'ampleur de la variation de la VAN est moins importante : elle double au lieu de tripler.

\section{Valeur des produits}

La modification de la valeur des produits engendre des variations non négligeables de rentabilité à l'hectare. D'ailleurs, on remarque que les rapports de sensibilité présentés au Tableau 9 sont très élevés comparativement à ceux obtenus pour tous les autres paramètres étudiés mis à part le coût des interventions. Une simple variation de $1 \%$ de la valeur des produits engendre des variations de 55 à $75 \$$ à l'hectare. Cette constatation nous amène à conclure que la valeur des bois constitue un paramètre très sensible. L'analyse des écarts des rapports entre les deux scénarios permet également d'affirmer que la valeur des produits constitue un paramètre plus sensible dans le cas du SCPE que dans le cas du SCJP. Encore une fois, la présence de flux monétaires importants dans le SCPE en tout début d'horizon explique cette situation.

\section{Coût des interventions}

L'estimation de la VAN des deux scénarios est davantage influencée par le coût des interventions que tout autre paramètre. La variation de la VAN plus importante dans le cas du SCPE (Tableau 7), de même qu'un rapport de sensibilité plus fort dans le cas du SCPE (Tableau 9), démontrent que le coût des interventions est plus sensible pour ce scénario. La coupe progressive d'ensemencement arrivant en début d'horizon et étant le traitement le plus coûteux parmi ceux retenus dans le cadre du projet, il est donc normal que ce scénario soit plus sensible à une variation des coûts, si minime soit-elle. Dans une étude similaire conduite au Brésil, une analyse de sensibilité a aussi démontré que la VAN de deux scénarios évalués sur des rotations de 35 ans était plus sensible à la valeur des produits et aux coûts de récolte qu'à l'efficience des opérations et aux rendements de croissance dus à l'application de traitements sylvicoles différents (Boltz et al. 2001).

\section{Conclusion}

Cette étude a permis d'élaborer une approche permettant de comparer deux scénarios sylvicoles contrastés dans une strate où différentes options sont envisageables. Devant un manque d'information à long terme sur les impacts de la sylviculture en peuplements mixtes, il a été décidé de travailler avec les outils et les hypothèses d'aménagement utilisés pour cette strate dans la pratique courante. Il est bien entendu que ceuxci comportent une grande imprécision. Notre démarche demeure toutefois cohérente avec celle utilisée pour les calculs de possibilité. L'analyse de sensibilité a été retenue pour éta l'impact de cette imprécision et identifier des pistes permettant d'améliorer le calcul de rentabilité. L'analyse financière a été réalisée en tenant compte du point de vue du gestionnaire de la forêt. Même si l'application a été faite sur un territoire de forêt publique, nous croyons que la démarche pourrait être reproduite en forêt privée.

L'analyse financière effectuée avec une strate d'aménagement constituée majoritairement de bouleaux jaunes et de résineux a permis de constater que, de façon générale, le SCJP est légèrement plus rentable financièrement, bien qu'il soit plus sensible aux variations de certains paramètres que le SCPE. Devant le faible écart de rentabilité, des arguments d'ordre écologique ou social pourraient contribuer à favoriser l'un ou l'autre scénario.

Dans une perspective de gestion du risque en aménagement forestier, il devient important de connaitre quels paramètres influencent le plus la rentabilité du scénario envisagé. Une attention particulière devrait être portée à établir avec précision la valeur des produits et le coût des opérations puisque la valeur actuelle nette des deux scénarios est davantage sensible à ces deux paramètres plutôt qu'aux autres éléments considérés. Dans le cas du SCPE, nous savons que le raffinement des méthodes de récolte dans les prochaines années risque fortement de réduire les coûts associés à la coupe d'ensemencement. Le coût de ce traitement est présentement élevé en raison des normes actuelles qui exigent un martelage positif des semenciers suivi d'un martelage négatif des tiges à éliminer en plus du caractère expérimental de l'étude utilisée. Les résultats de l'analyse de sensibilité démontrent alors que nous avons tout intérêt à investir pour diminuer le coût de la coupe d'ensemencement. Le scénario d'évolution s'avère être le paramètre le moins sensible. Une incertitude quant à la composition des peuplements futurs ne semble donc pas source de risque. La matrice de répartition par produit constitue finalement le paramètre sylvicole le plus sensible. Il devient donc important de connaitre l'impact de la sylviculture sur la qualité des tiges produites, de même que la qualité des tiges pouvant être prélevées de la forêt au cours d'une intervention.

Puisque l'analyse de sensibilité ne porte que sur les résultats d'une seule strate avec comme hypothèses de départ les rendements prévus au MAF, il faut rester prudent quant aux conclusions à tirer. Le SCPE pourrait devenir intéressant si les taux d'accroissement étaient inférieurs à ceux anticipés. Dans le cadre d'un scénario où la sylviculture intensive permettrait d'améliorer la qualité des produits obtenus, il serait plus rentable d'opter pour le SCPE, bien qu'un peu plus risqué. Finalement, malgré que le SCPE soit plus sensible aux variations des paramètres financiers, le SCJP demeure pratiquement toujours plus rentable, indépendamment des variations négatives ou positives effectuées.

\section{Remerciements}

Nous tenons à remercier Commonwealth Plywood Co. Ltd. et Christian Picard de nous avoir fourni les données sylvicoles et financières nécessaires à la réalisation de cette étude. Nos 
remerciements vont également au MRNF pour le support financier et l'aide technique, notamment Marie-Kim Savoie et Michel Letarte. Nous tenons à souligner l'aide de Guy Lessard, Donald Blouin, Michel Huot et Pierre Gastaldello pour la participation de près ou de loin à la revue de littérature.

\section{Références}

Anderson, H.W., B.D. Batchelor, C.M. Corbett, A.S. Corlett, D.T. Deugo, C.F. Husk et W.R. Wilson. 1990. A silvicultural guide for the tolerant hardwoods working group in Ontario. Ont. Min. Nat. Res., Science and Technology Series vol. 7.

Archambault, L., J. Morissette et M. Bernier-Cardou. 1997. Forest succession over a 20-year period following clearcutting in balsam fir-yellow birch ecosystems of eastern Québec, Canada. For. Ecol. Manage.102: 61-74.

Bédard, S. et F. Brassard. 2002. Les effets réels des coupes de jardinage dans les forêts publiques du Québec en 1995 et 1996. Ministère des Ressources naturelles, Québec. 25 p.

Boivin, J.-L. 1971. Étude de la régénération après coupe rase dans des peuplements feuillus et mélangés de l'ouest québécois. For. Chron. $47: 82-85$.

Boltz, F., D.R. Carter, T.P. Holmes et R. Pereira. 2001. Financial returns under uncertainty for conventional and reduced-impact logging in permanent forests of the Brazilian Amazon. Ecological Economics 39 : 387-398.

Davis, L.S. et K.N. Johnson. 1987. Forest Management. 3e ed. McGraw-Hill. New York. 790 p.

Del Degan, Massé et Associés. 2002. Enquête sur les coûts de la sylviculture en forêt publique : 2000-2001. Rapport final. Présenté au ministère des Ressources naturelles. Disponible au http://www. mrnfp.gouv.qc.ca/publications/forets/entreprises/enquete_coutsrapport.pdf [consulté le 30 mai 2003].

Erdmann, G.G. 1990. Betula alleghaniensis Britton - Yellow Birch. Dans R.M. Burns et B.H. Honkala (tech. coords.). Silvics of North America Vol. 2. Hardwoods. pp. 133-147. Agriculture Handbook 654. U.S. Dept. of Agriculture, Forest Service, Washington, DC.

Erdmann, G.G., R.M. Peterson Jr. et R.M. Godman. 1981. Cleaning yellow birch seedlings stands to increase survival, growth and crown development. Can. J. For. Res.11 : 62-68.

Godbout, C. 2002. Le problème de l'éclaircie commerciale des bétulaies blanches de 60 ans et plus. Revue de littérature. Note de recherche forestière No 115. Ministère des Ressources naturelles, Direction de la recherche forestière, Sainte-Foy, QC. 12 p.

Gregersen, H.M. et A.H. Contreras. 1980. Analyse économique des projets forestiers. FAO, Organisation des nations unies pour l'alimentation et l'agriculture, Rome. Étude no. 17.214 p.

Grondin, P., C. Ansseau, C. Bélanger, J.F. Bergeron, Y. Bergeron, G. Lessard, S. Payette, P.J.H. Richard, J.P. Saucier, L. Sirois et L. Vasseur. 1996. Écologie forestière. Dans Manuel de Foresterie. Ordre des Ingénieurs forestiers du Québec. pp.133-279. Presses de l'Université Laval, Sainte-Foy, QC.

Hanewinkel, M. 2001. Economic aspects of the transformation from even-aged pure stands of Norway spruce to uneven-aged mixed stands of Norway spruce and beech. For. Ecol. Manage.151 : 181-193.

Hannah, P.R. 1974. Thinning yellow birch saplings to increase main leader growth. Vermont Agricultural Experiment Station. Research paper MP 80. University of Vermont, Burlington. 9 p.

Hannah, P.R. 1988. The shelterwood method in northeastern forest type: A literature review. NJAF 5: 70-77.

Kelty, M.J. et R.D. Nyland. 1981. Regenerating Adirondack northern hardwoods by shelterwood cutting and control of deer density. J. For. 79 : 22-26.

Klemperer, W.D. 1996. Forest resources economics and finance. McGraw-Hill. Inc., New York, Montréal. 551 p.
Knoke, T. et N. Plusczyk. 2001. On economic consequences of transformation of a spruce (Picea abies (L.) Karst.) dominated stand from regular into irregular age structure. For. Ecol. Manage. 151 : 163-179. Larocque, G. 1985. Regeneration in a tolerant hardwood stand managed under single-tree selection. Can. For. Serv., Petawawa Nat. For. Inst. Inf. Rep. PI-X-50.

Leak, W.B., D.S. Solomon et P.S. Debald. 1986. Silvicultural guide for Northern Hardwood types in the Northeast (revised). Res. Pap. NE-603. USDA For. Ser. Northeast. For. Exp. Stn. Upper Darby, PA. Lessard, G., S. Bédard et F. Pelletier. 2004. Méthode de calcul de la possibilité forestière avec SYLVA II. $15^{\mathrm{e}}$ version. Huit chapitres et annexes. CERFO. Sainte-Foy, QC. 210 p.

Malenfant, A. et A. Patry. 2002. Comparaison de différents traitements sylvicoles pour l'installation de la régénération en bouleau jaune dans des peuplements mixtes à dominance feuillue - Suivi 2001. Groupement forestier Baie-des-Chaleurs. New Richmond, QC. 73 p. Marquis, D.A. 1967. Clearcutting in Northern Hardwoods: Results after 30 Years. Northeastern Forest Experiment Station, Upper Darby, PA. U.S. Forest Service Research Paper NE-85. 13 p.

Meunier, S., G. Gravel, G. Lessard, D. Blouin et A. Patry. 2002. Étude sur les traitements sylvicoles favorisant la régénération du bouleau jaune à la station forestière de Duchesnay. Rapport final 3401-al30/01/03. 29 p.

Ministère des Ressources naturelles (MRN). 1998. Manuel d'aménagement forestier, 3ème édition, Direction des programmes forestiers du ministère des Ressources naturelles. 122 p. et annexe. .1999. Manuel de mise en valeur des forêts privées du Québec, document d'annexes. Direction des communications du Ministère des Ressources naturelles. 180 p. et annexe.

2002. Instruction relatives à l'application du règlement sur la valeur des traitements sylvicoles admissibles en paiement de droit de coupe - Exercice 2003-04. Forêt Québec. Direction de l'assistance technique. Division des traitements sylvicoles. 88 p. + annexes.

Morissette, D. 1994. Décisions financières à long terme. Les Éditions SMG. Trois-Rivières, QC. 545 p.

Niese, J.N. et T.F. Strong. 1992. Economic and tree diversity trade-offs in managed northern hardwoods. Can. J. For. Res. 22 : 1807-1813.

Niese, J.N., T.F. Strong et G.G. Erdmann. 1995. Forty years of alternative management practices in second-growth, pole-size northern hardwoods. II. Economic evaluation. Can. J. For. Res. 25 : $1180-1188$.

Nyland, R.D. 1996. Silviculture. Concepts and applications. McGraw Hill, New York. 633 p.

Perala, D.A. et A.A. Alm. 1990. Regeneration silviculture of birch: A review. For. Ecol. Manage. 32 : 39-77.

Roberge, M.R. 1977. Results of research in northern hardwood silviculture at Dudswell. For. Chron. 53 : 223-225.

Roberge, M.R. 1979. La coupe à blanc par bandes peut-elle maintenir les strates actuelles comme strates de retour? Progrès Forestier (Déc) : 42-43.

Syndicat des producteurs de bois de la Mauricie (SPBM). 2003. Info-Sciage. Forêts nouvelles. Bulletin d'information des producteurs de bois de la Mauricie. 17(3) : 8-11.

Syndicat des propriétaires forestiers de la région de Québec (SPFRQ). 2003. Prix du bois destiné au sciage, déroulage, aux pâtes et papiers, panneaux et autres produits [en ligne]. http://www.spfrq. qc.ca/Prix/ [consulté le 22 mai 2002].

Tubbs, C.H. 1987. The cutting edge. Dans R.D. Nyland (ed.). Managing northern hardwoods, Proc. of a Silvicultural Symposium. pp. 412-415. State University of New York, Syracuse, NY.

Tubbs, C.H. et F.T. Metzger. 1969. Regeneration of Northern hardwoods under shelterwood cutting. For. Chron. 45 : 333-337.

Walters, R.S. et R.D. Nyland. 1989. Clearcutting Central New York Northern Hardwood Stands. NJAF 6 : 75-78.

Weitzman, M.L. 2001. Gamma Discounting. The American Economic Review 91(1) : 260-271. 